\title{
Determination of structures from defined nanocrystalline regions by scanning nanobeam diffraction tomography
}

Authors: Marcus Gallagher-Jones ${ }^{\mathrm{a}}$, Karen C. Bustillo ${ }^{\mathrm{b}}$, Colin Ophus ${ }^{\mathrm{b}}$, Jim Ciston ${ }^{\mathrm{b}}$, Andrew M. Minor $^{\mathrm{b}, \mathrm{c}}$, and Jose A. Rodriguez ${ }^{\mathrm{a}}$

aDepartment of Chemistry and Biochemistry, UCLA-DOE Institute for Genomics and Proteomics, University of California Los Angeles, Los Angeles, CA 90095, USA. marcusgj@chem.ucla.edu, irodriguez@chem.ucla.edu

${ }^{b}$ National Center for Electron Microscopy, Molecular Foundry, Lawrence Berkeley National Laboratory, CA, USA. kbustillo@lbl.gov, clophus@lbl.gov, jciston@lbl.gov

'Department of Materials Science and Engineering, University of California Berkeley, CA, USA. aminor@berkeley.edu

Crystallography is intrinsically limited by its reliance on signal averaged over large collections of perfectly ordered molecules within a single crystal lattice or even across multiple crystals. This is limiting when crystals contain severe disorder pathologies or are beam sensitive. Recent advances in electron diffraction have reduced the minimum size of crystals useful for structure determination to $100 \mathrm{~s}$ of $\mathrm{nms}^{1-3}$, overcoming many of these limitations. Scanning nano-beam diffraction has been applied to the study of nanomosaicity within a single micron-scale crystal ${ }^{4}$, illustrating the benefit of scanning nano-beams for structure determination. We demonstrate that scanning a sub-10nm electron probe over a peptide nanocrystal at multiple orientations yields a collection of patterns that represent a full tilt series across reciprocal space. Use of a direct electron detector allows meaningful intensities to be extracted from sparse, individual diffraction patterns; this reduced data is sufficient for phasing by molecular replacement and compares favorably with data collected by selected area diffraction. Scanning nanobeam diffraction can be collected from regions of a crystal as small as $40 \mathrm{~nm}$, allowing for the fine sampling of specific areas within a single crystal. This approach has implications for circumventing polycrystallinity, reducing radiation damage and accessing the ensemble of structures present within a crystal.

\section{References}

1. Lanza, A. et al. Nanobeam precession-assisted 3D electron diffraction reveals a new polymorph of hen egg-white lysozyme. IUCrJ 6, (2019).

2. Mugnaioli, E. et al. Ab Initio Structure Determination of $\mathrm{Cu} 2-\mathrm{x}$ Te Plasmonic Nanocrystals by Precession-Assisted Electron Diffraction Tomography and HAADF-STEM Imaging. Inorganic chemistry 57, 10241-10248 (2018).

3. Sawaya, M. R. et al. Ab initio structure determination from prion nanocrystals at atomic resolution by MicroED. PNAS 113, 11232-11236 (2016).

4. Gallagher-Jones, M. et al. Nanoscale mosaicity revealed in peptide microcrystals by scanning electron nanodiffraction. Communications Biology 2, 26 (2019). 